Research, Society and Development, v. 9, n. 8, e910986254, 2020

(CC BY 4.0) | ISSN 2525-3409 | DOI: http://dx.doi.org/10.33448/rsd-v9i8.6254

\title{
Segurança Alimentar à luz de novas ferramentas educativas na pedagogia crítica
}

Food Security in the light of new educational tools in critical pedagogy

Seguridad Alimentaria a la luz de nuevas herramientas educativas en pedagogía crítica

Recebido: 02/07/2020 | Revisado: 15/07/2020 | Aceito: 20/07/2020 | Publicado: 02/08/2020

\section{Érica Silva Gonçalves Cabral}

ORCID: https://orcid.org/0000-0002-5372-8711

Instituto Federal do Rio Grande do Norte, Brasil

E-mail: ericasgcabral@gmail.com

Luís Gomes de Moura Neto

ORCID: https://orcid.org/0000-0002-2318-4637

Instituto Federal de Pernambuco, Brasil

E-mail: luisgomesmn@gmail.com

\section{Resumo}

No intuito de garantir a produção e o consumo de alimentos seguros, medidas higiênicosanitárias devem ser desenvolvidas e aplicadas em todas as etapas da cadeia alimentar. A legislação, desenvolvida ao longo da história humana, preconiza as condições de qualidade necessárias para que um alimento possa ser ingerido sem causar danos à saúde das pessoas. Sendo assim, como forma de auxiliar o processo de ensino-aprendizagem sobre o tema, o objetivo deste presente artigo é abordar conceitos, fatores e aspectos relativos à Segurança Alimentar através da perspectiva de uma tendência pedagógica crítica social de conteúdos ou histórico-crítica, valorizando as novas ferramentas educacionais. Para isso, a metodologia empregada foi a realização de uma pesquisa de natureza básica, caracterizada como bibliográfica e exploratória. Contudo, o conhecimento crítico e consciente sobre os perigos biológicos, químicos e físicos aos quais os alimentos estão submetidos e quanto à ação humana desordenada no meio ambiente são importantes requisitos para que todos os organismos institucionais e a sociedade como um todo possam se posicionar e exigir que a Segurança Alimentar exista cada vez mais concretamente na vida das pessoas. E as novas ferramentas educativas podem auxiliar no processo de construção deste saber.

Palavras-chave: Segurança alimentar; Pedagogia crítica; Novas ferramentas educacionais. 


\section{Abstract}

In order to ensure the production and consumption of safe food, hygienic-sanitary measures should be developed and applied at all stages of the food chain. Legislation, developed throughout human history, advocates the quality conditions necessary for a food to be ingested without causing harm to people's health. Therefore, as a way of assisting the teaching-learning process on the subject, the objective of this present article is to approach concepts, factors and aspects related to food security through the perspective of a critical pedagogical social trend of contents or historical-critical. For this, the methodology used was to carry out a basic research, characterized as bibliographic and exploratory. However, critical and conscious knowledge of the biological, chemical and physical dangers to which food is subjected and of disordered human action in the environment are important requirements for all institutional bodies and society as a whole to position themselves and demand that Food Security exists more and more concretely in people's lives. And the new educational tools can assist in the process of building this knowledge.

Keywords: Food security; Critical pedagogical; New educational tools.

\section{Resumen}

Para garantizar la producción y el consumo de alimentos inocuos, se deben desarrollar y aplicar medidas higiénico-sanitarias en todas las etapas de la cadena alimentaria. La legislación, desarrollada a lo largo de la historia humana, recomienda las condiciones de calidad necesarias para que se pueda comer un alimento sin dañar la salud de las personas. Por lo tanto, como una forma de ayudar al proceso de enseñanza-aprendizaje sobre el tema, el objetivo de este artículo es abordar conceptos, factores y aspectos relacionados con la Seguridad Alimentaria através de la perspectiva de una tendencia pedagógica social crítica o histórico-crítica, valorando nuevas herramientas educativas. Para ello, la metodología utilizada fue realizar una investigación básica, caracterizada como bibliográfica y exploratoria. Sin embargo, el conocimiento crítico y consciente de los riesgos biológicos, químicos y físicos a los que están sometidos los alimentos y de la acción humana desordenada en el medio ambiente son requisitos importantes para que todos los organismos institucionales y la sociedad en su conjunto se posicionen y exijan que La Seguridad Alimentaria existe cada vez más concretamente en la vida de las personas. Y las nuevas herramientas educativas pueden ayudar en el proceso de construcción de este conocimiento.

Palabras clave: Seguridad alimentaria; Pedagogía crítica; Nuevas herramientas educativas. 


\section{Introdução}

O Codex Alimentarius, 2006, define higiene dos alimentos como sendo "todas as condições e medidas necessárias para garantir a segurança e a adequação dos alimentos em todas as etapas da cadeia de alimentos".

Em 1967, na primeira legislação de alimentos desenvolvida, um dos elementos componentes do padrão de identidade e qualidade de um alimento referia-se à fixação de critérios de qualidade segundo a norma e especificação atribuída. A expressão controle de qualidade corresponde à manutenção dos produtos e serviços dentro dos níveis de tolerância aceitáveis para o consumidor direto ou comprador. Assim, para avaliar a qualidade de um produto alimentício, deve ser mensurado o grau em que o produto satisfaz os requisitos específicos (Cavalli e Salay, 2001).

Caracterizam-se como atividades de controle da qualidade as análises físico-químicas, sensoriais e microbiológicas. Contudo, o controle da qualidade deve ser praticado de forma contínua, e não apenas no produto final, com vias a oferecer maior garantia aos usuários do serviço, o que viabiliza aumento da confiabilidade por parte dos consumidores e minimização dos riscos à saúde (Marins et al, 2014).

Os avanços tecnológicos disponíveis hoje e que atestam a qualidade higiênicosanitária dos alimentos foram baseados na história construída entre o homem e a forma de obtenção dos alimentos. Com a descrição do processo histórico e a organização das práticas constituídas, Rosen (1994 apud Costa, 2004, p. 34) enuncia que as praças das cidades medievais destacavam-se porque nelas coexistiam várias faces da sociedade, como, por exemplo, o comércio, inclusive de alimentos; as reuniões sociais, religiosas e artísticas; as cerimônias e mesmo conspirações. Neste sentido, os mercados e praças tornaram-se objeto de proteção da saúde da coletividade, com base em noções que permitiram relacionar o aparecimento de doenças com o consumo de comida estragada ou deteriorada.

Surge, assim, uma medida cautelar de proteção à saúde do consumidor local proposta pelas autoridades. Nessa perspectiva, e com a organização da vida em sociedade, a saúde passa a ser considerada um dos direitos fundamentais do ser humano, sem distinção de raça, religião, classe social ou econômica. Com os avanços das normas legais para a defesa da vida dos indivíduos, a Organização Mundial da Saúde também buscou promulgar atividades no intuito de orientar os Estados a desenvolverem ações no campo da medicina preventiva, de caráter individual e coletivo, e para a recuperação e a promoção da saúde (Marins et al, 2014). 
Diante desta perspectiva, este artigo tem sua razão de ser porque busca valorizar uma proposta pedagógica histórico-crítica que favoreça o processo de ensino-aprendizagem sobre aspectos relativos à Segurança Alimentar.

Como forma de auxiliar o processo de ensino-aprendizagem sobre o tema, o objetivo deste presente artigo é abordar conceitos, fatores e aspectos relativos à Segurança Alimentar através da perspectiva de uma tendência pedagógica crítica social de conteúdos ou históricocrítica, valorizando as novas ferramentas educacionais.

\section{Metodologia}

Consiste o método em um modo para se alcançar determinado fim, implicando na escolha de uma concepção da realidade ou de um fragmento da mesma como objeto de estudo e, a partir daí, iniciam-se os procedimentos metodológicos para, segundo Riquena, 2013, explicar minuciosa, detalhada, rigorosa e exatamente toda a ação desenvolvida e as suas etapas.

Para o objeto de estudo propõe-se, para a relação entre o processo de ensino e aprendizagem e o uso de uma ferramenta educacional auxiliar ao mesmo, o desenvolvimento de uma prática educativa pedagógica histórico-crítica para abordar sobre aspectos da segurança alimentar. Neste intuito, foi realizada uma pesquisa bibliográfica, visando o levantamento de dados sobre tal temática de interesse coletivo em plataforma digital, por exemplo, como o Google.

Também há característica de pesquisa básica pois corresponde a um levantamento para se obter mais conhecimento científico acerca de um tema. Difere da pesquisa aplicada e é conhecida como pura ou fundamental (Gerhardt e Silveira, 2009).

$\mathrm{Na}$ mesma intenção de realizar um estudo para adquirir mais familiaridade com um fenômeno social ou outro que está sendo investigado, faz-se uma pesquisa exploratória, possuindo mais flexibilidade qualitativa no momento da obtenção dos dados (Gerhardt e Silveira, 2009).

\section{Alimentos Seguros e Segurança Alimentar}

De acordo com os Princípios Gerais de Higiene Alimentar do Codex Alimentarius (Food and Agriculture Organization of the United Nations, 1998), o alimento seguro é aquele que, ao ser consumido, não produzirá danos à saúde dos consumidores. Dessa 
forma, a segurança alimentar engloba práticas que garantem a qualidade do alimento, desde a sua pré-produção até o seu consumo final, para que ele não apresente riscos de naturezas variadas à saúde do consumidor.

Uma das naturezas destes riscos corresponde a perigos biológicos, que implicam no grau de segurança dos alimentos, assim como microrganismos patogênicos, suas toxinas e os insetos, podendo ocasionar um problema para a agricultura por conta do hábito alimentar e comportamento de algumas espécies; do potencial biótico elevado, ou seja, da capacidade reprodutiva das mesmas; e da ação humana na mudança do meio ambiente, provocando um desequilíbrio ecológico e permitindo a propagação das espécies com as duas características anteriormente descritas (Neves, 1995).

Esta ação humana refere-se ao desmatamento, a monoculturas, à criação intensiva de animais, à superpopulação de pessoas, a condições inadequadas de escoamento de águas servidas e de remoção de dejetos e do lixo produzido, a precárias condições de higiene, vestuário, moradia e alimentação, levando à construção de sociedades predatórias e desorganizadas socioeconomicamente, que criam um ambiente propício para a reprodução em larga escala de pragas (Neves, 1995).

Os inseticidas utilizados na agricultura ou agropecuária podem representar, por sua vez, perigos químicos aos alimentos em produção, podendo conferir caráter impróprio aos mesmos para o consumo humano. Um livro chamado Primavera Silenciosa, escrito em 1962 pela jornalista americana Rachel Carson, alertou o grande público para o sério problema do uso indiscriminado e incorreto de inseticidas, em condições de sub ou superdosagens, que pode favorecer efeitos maléficos (Neves, 1995).

Sanitizantes usados no preparo de alimentos, bem como metais pesados, também podem oferecer riscos químicos devido aos resíduos que podem deixar na comida a ser consumida. Já os perigos físicos são relacionados ao desprendimento de metais, vidros, plásticos, borrachas, areia, parafusos e outros materiais existentes nas embalagens ou nas máquinas durante o processamento dos alimentos (Food and Agriculture Organization of the United Nations, 1998).

Na década de 1970, época de crise de alimentação mundial, o termo segurança alimentar surgiu para direcionar ações quanto a problemas de abastecimento de alimentos, ou seja, de como garantir a disponibilidade e, de certo modo, a estabilidade dos preços dos alimentos básicos em nível internacional e nacional (Food and Agriculture Organization of the United Nations, 2003). 


\subsection{Fatores que interferem na Segurança Alimentar devido aos seus impactos ambientais}

A produção de alimentos pela agricultura, do modo simples ao mais complexo, sempre implica desequilíbrio para o meio natural, pois, cultivar de forma concentrada, pode tornar a planta mais vulnerável ao ataque de insetos e às doenças, bem como, retirar a planta de nichos nos quais se encontra adaptada à umidade, ao solo e às condições climáticas significa alterar as suas propriedades intrínsecas. Com a sua disposição em outro local, ela muda a paisagem e altera a recepção de energia solar, do vento, do regime da água no solo e da química do solo ou seja, altera a biodiversidade, destruindo outros tipos de vida que dependiam do equilíbrio anterior (Porto-Gonçalvez, 2011).

A monocultura potencializou esses problemas e, ao longo do tempo, com o aumento de sua escala, foi possível ver as grandes modificações que causou ao meio ambiente, em geral produzindo mazelas de variadas formas para os humanos. A continuidade da produção de alimentos envolve um cuidado atencioso da natureza, o que pressupõe empenho em conhecê-la (Marins et al, 2014).

Porém, ainda que catástrofes ambientais, como secas e inundações, possam alterar o ritmo da produção de alimentos e produzir fome - mesmo que passageira -, é visível, hoje em dia, que a deterioração da terra e sua escassez, além de dependerem do crescimento populacional, estão acopladas ao modo de produção capitalista, centralizado no lucro, independentemente da qualidade do alimento. A procura por maiores quantidades de produtos tem feito com que a qualidade dos alimentos seja invariavelmente degradada, sobretudo pelo grande uso de produtos químicos na sua produção (Marins et al, 2014).

A monocultura, o uso de agrotóxicos e a intensa mecanização da agricultura são os meios empregados pelo agronegócio para obter maior produtividade de alimentos, atualmente. O processo de acumulação do capital foi gerando processos produtivos cada vez mais tecnificados para revalorizar e incrementar a taxa de mais-valia do capital. Com a expansão do capital em escala mundial e a abertura de novos campos de investimento, esta tecnoestrutura foi-se transferindo para os países "subdesenvolvidos". Este processo gerou uma organização produtiva dependente, que degradou a capacidade produtiva dos ecossistemas tropicais e a riqueza potencial de suas populações. Por sua vez, isto implicou a apropriação destes recursos pelas grandes potências industriais e uma distribuição cada vez mais desigual da riqueza produzida nos países assim explorados (Leff, 2009).

A questão dos alimentos e da alimentação saudável está se tornando um tema de debate generalizado, a ponto de grandes empresas multinacionais do setor revisarem suas 
(CC BY 4.0) | ISSN 2525-3409 | DOI: http://dx.doi.org/10.33448/rsd-v9i8.6254

estratégias de negócios para produzirem com impacto menor sobre o meio ambiente e com melhores índices de nutrientes e menores quantidades de gordura, sal e açúcar, por exemplo (Nitzke, 2012).

Entretanto, em tempos de aquecimento global, outro aspecto que vem gerando cada vez mais discussões é o impacto dos processos de produção, distribuição, comercialização e preparo de alimentos no consumo de energia. Baseado neste fato, cresce, nos Estados Unidos e na Europa, um movimento de valorização da "comida local", ou seja, de estímulo ao consumo de produtos - até mesmo industrializados - obtidos com insumos da região onde vivem os consumidores e na qual tenham a mínima necessidade possível de deslocamento entre a produção e o consumo (Nitzke, 2012).

\section{Tendência Pedagógica Crítica como Proposta para Prática Educativa sobre Aspectos da Segurança Alimentar}

Das tendências pedagógicas mais dominantes em nosso meio de atuação, como a pedagogia tradicional, a renovada, por condicionamento e a de caráter progressista, como a libertadora, a libertária e a histórico-crítica, a partir dos princípios, métodos e consequências ao nível individual e social de cada pedagogia mencionada, a crítica social pode produzir melhores resultados que as demais correntes pedagógicas, considerando ressaltar a participação ativa e a criticidade de todos os sujeitos envolvidos nos processos de ensinoaprendizagem, bem como a necessidade de transformação da realidade social em que eles vivem (Pereira, 2003).

No entanto, não há um processo educacional asséptico de ideologias dominantes, sendo necessária a reflexão sobre o próprio sentido e valor da educação na e para a sociedade. Educar, conforme Saviani, 1985, e Luckesi, 1994, não significa simplesmente transmitir e/ou adquirir conhecimentos. No processo educativo, existe uma série de representações de sociedade e de homem que se quer formar. Através da educação, as novas gerações apropriam-se dos valores culturais e reproduzem e/ou transformam os códigos sociais.

No Relatório Final da XI Conferência Nacional de Saúde (CNS, 2000:165-166), no capítulo sobre a Democratização das Informações, tem-se que:

"As políticas de IEC (Informação, Educação e Comunicação) devem estar voltadas para a promoção da saúde, que abrange a prevenção de doenças, a educação para a saúde, a proteção da vida, a assistência curativa e a reabilitação sob responsabilidade das três esferas de governo, utilizando pedagogia crítica, que leve o usuário a ter 
conhecimento também de seus direitos; dar visibilidade à oferta de serviços e ações de saúde do SUS; motivar os cidadãos a exercer os seus direitos e cobrar as responsabilidades dos gestores públicos e dos prestadores de serviços de saúde".

Do objetivo principal de nortear a condução docente, moldando-a a partir das necessidades de ensino observadas no âmbito social em que vivia, as tendências pedagógicas foram concebidas com base nas visões em relação ao contexto histórico das sociedades, abrindo um leque de possibilidades de direcionamento do trabalho dos responsáveis por educar a partir de suas convicções pessoais, profissionais, políticas e sociais, contribuindo para a produção de uma prática pedagógica significativa, esclarecedora e, principalmente, interessante para os educandos, motivando as pessoas envolvidas no processo a sonharem, pensarem e criarem (Santos, 2012; Gadotti, 2000).

Em Santos, 2012, como afirma Libâneo, aprender é um ato de conhecimento da realidade concreta, isto é, da situação real vivida pelas pessoas que buscam aprendizados, e só tem sentido se resulta de uma aproximação crítica dessa realidade, o que está em consonância com o que diz Saviani (1991):

a Pedagogia Crítica implica a clareza dos determinantes sociais da educação, a compreensão do grau em que as contradições da sociedade marcam a educação e, consequentemente, como é preciso se posicionar diante dessas contradições e desenredar a educação das visões ambíguas para perceber claramente qual é a direção que cabe imprimir à questão educacional (p. 103).

\subsection{Novas Ferramentas Educacionais}

O desenvolvimento de produtos educacionais, a implementação deles em sala-de-aula e um relato desta experiência de implementá-los é o objetivo do Mestrado Profissional ligado ao Ensino. A tentativa é no intuito de levar os resultados das pesquisas acadêmicas em ensino, já executadas há alguns anos, para além das revistas científicas e para a prática docente do dia-a-dia. Um aplicativo, um texto formativo docente, um vídeo, uma estratégia didática, o uso do computador e smartphone, um jogo, uma oficina, um minicurso, dentre outros, produzidos e aplicados em sala de aula para ensinar, consistem em exemplos de produtos educacionais (Moreira, 2018).

As possibilidades são inúmeras, sendo importante seguir os passos da inovação na feitura do produto, da geração do produto propriamente dito após seu planejamento, do seu uso aplicado em situação real de aula e do registro do relato dissertativo de como se deu a 
experiência e o que aconteceu de resultado com a mesma. Para isso, é aconselhável ter acesso a relatos de experiência parecidas na literatura acadêmica e científica, bem como tentar adquirir alguma visão do que é ensinar e do que é testar uma ferramenta educacional. A dissertação do Mestrado Profissional deve ser um relato de experiência bem feito, com foco no desenvolvimento do produto e na sua utilização nos ambientes educativos formais ou não (Moreira, 2018).

A rotina de trabalho, necessidades específicas de elaboração, acervo de conhecimentos teóricos e práticos, cuidados técnicos na execução, intuição e criatividade, além de adaptação entre cada item ligado à Informática, Educação e Comunicação Social, bases sobre as quais o professor poderá planejar a organização de seu conteúdo, para ser acessado segundo os moldes de cultura cognitiva do aluno do Século XXI, educado (e portanto exigente), norteiam reflexões sobre a natureza da tecnologia de educação informatizada (Timm et al, 2003).

Como na elaboração de um programa de TV ou em uma chamada ao vivo para narrar uma catástrofe ou outro acontecimento histórico-crítico, a produção de um recurso audiovisual de massa ou de um jogo ou de uma atividade gamificada de qualidade envolve profissionais (como artistas, designers, programadores, professores - consultores pedagógicos e conteudistas) que trabalham para a realização do projeto e, portanto, demandam um custo (Tonéis, 2017).

A importância para o diálogo entre as disciplinas quando os produtos educacionais são formulados em um Mestrado Profissional, na perspectiva da interdisciplinaridade como uma atitude transformadora para a questão do conhecimento, deve ser dada e voltada a uma prática pedagógica diversa e em construção (Moreira; Rôças; Pereira; Anjos, 2017).

Segundo Ferreira, 2010, forma interdisciplinar é utilizada em associação à definição de Educação Ambiental retirada de base legal, consistindo na qualidade de vida sadia e sua sustentabilidade e enfatizando a interdisciplinaridade metodológica e epistemológica da mesma. Um texto de apoio pode trazer mais de uma representação para interdisciplinaridade, o que não ocasiona conflito entre as ideias apresentadas no produto educacional.

A Educação Permanente em Saúde, por exemplo, que está presente no cotidiano das organizações e do trabalho, pode ser entendida como uma atualização cotidiana das práticas com novos aportes teóricos, metodológicos, científicos e tecnológicos disponíveis, contribuindo para a construção de relações e processos que emergem do interior das equipes, com seus agentes e práticas organizacionais, incluindo as interinstitucionais e/ou intersetoriais (Leite e Lima, 2019).

A reflexão dos docentes sobre suas práticas, aliada à disposição para encontrar 
caminhos para promover a aprendizagem dos estudantes e à consulta a diferentes fontes, resultou em questionamentos que culminaram na elaboração dos produtos educacionais. Esses elementos produziram um conhecimento que foi aplicado em sala de aula, levando os docentes, nessa etapa, a aproximarem a pesquisa da prática docente e, assim, construírem saberes (Vital e Guerra, 2017).

Os alunos que trabalharem de modo ativo podem experimentar a possibilidade de crescerem pessoalmente e profissionalmente, desenvolvendo habilidades e competências. $\mathrm{O}$ limite para a evolução e desenvolvimento é o dos próprios alunos, que poderão aprender muito sem as travas e freios dos sistemas convencionais ou tradicionais (Pereira et al, 2018).

\section{Considerações Finais}

A Segurança Alimentar existe quando todos, em todas as ocasiões, têm acesso físico e socioeconômico a alimentos suficientes, seguros e nutritivos, satisfazendo suas necessidades alimentares para uma vida saudável, devendo ser realizada em bases sustentáveis e tratando de políticas públicas que garantam o acesso quantitativo e qualitativo das pessoas aos mesmos (Tibola e Santi, 2017; Marques et al, 2017).

O conhecimento crítico e consciente sobre os perigos biológicos, químicos e físicos aos quais os alimentos estão submetidos e quanto à ação humana desordenada no meio ambiente são importantes requisitos para que todos os organismos institucionais e a sociedade como um todo possam se posicionar e exigir que conceitos, aspectos e fatores que se relacionam à Segurança Alimentar sejam cada vez mais conhecidos e que a mesma exista concretamente na vida das pessoas.

A utilização de novas ferramentas educacionais, como um jogo interdisciplinar, por exemplo, no intuito de auxiliar no processo de ensino-aprendizagem, vem sendo empregada, especialmente com a elaboração de produtos educativos em Mestrado Profissional ligado ao Ensino. Deste modo, a temática de Segurança Alimentar também pode ser abordada através de um destes recursos educativos, visando melhorar os níveis de concentração, memória e associação de ideias nos alunos sobre este assunto que precisam conhecer.

Contudo, mais estudos, projetos e trabalhos ligados ao tema merecem ser abordados e desenvolvidos para fomentar o saber científico e proporcionar que a teoria possa ser mais praticada no âmbito das organizações trabalhistas e das instituições de ensino, bem como no dia-a-dia dos cidadãos. 


\section{Referências}

Cavalli, S. B., \& Salay, E. (2001). Segurança do alimento e recursos humanos: estudo exploratório em restaurantes comerciais dos municípios de Campinas, SP e Porto Alegre, RS. Higiene Alimentar, São Paulo, 18(126-127), 29-35.

Conselho Nacional de Saúde. (2000). 11 a Conferência Nacional de Saúde: Efetivando o SUS: Acesso, Qualidade e Humanização na atenção à Saúde, com Controle Social Relatório Final. Recuperado de <http://conselho.saude.gov.br/ 11 conferencia/ anexos/relatorio.pdf.>.

Codex Alimentarius. (2006). Higiene dos alimentos: textos básicos. Brasília: Organização Pan-Americana da Saúde.

Costa, E. A. (2000). Conceitos e área de abrangência. In: Rozenfeld, S. (org.). Fundamentos de vigilância sanitária. Rio de Janeiro: Editora Fiocruz, 41-48.

Food and Agriculture Organization of the United Nations. (1998). Section 2 - Recommended International Code of Practice - General Principles of Food Hygiene. In: FAO. Food Quality and Safety Systems - A Training Manual on Food Hygiene and the Hazard Analysis and Critical Control Point (HACCP) System.

Food and Agriculture Organization of the United Nations. (2003). Trade Reforms and Food Security. Chapter 2. Food Security: Concepts and Measurement.

Ferreira, C. F. B. (2010). Formação de professores: concepções e práticas pedagógicas de Educação Ambiental. Nilópolis: IFRJ.

Gadotti, M. (2000). Revista Nova Escola.

Gerhardt, T. E., \& Silveira, D. T. (2009). Acesso 21 de novembro de 2018, Recuperado de http://www.ufrgs.br/cursopgdr/downloadsSerie/derad005.pdf

Leff, H. (2009). Ecologia, capital e cultura: a territorialização da racionalidade ambiental. Petrópolis: Vozes. 
Leite, I. C. M., \& Lima, R. R. T. de. (2019). Educação permanente em saúde: uma possibilidade para aperfeiçoar o trabalho em uma Escola Técnica do Sistema Único de Saúde? Research, Society and Development, 9(3).

Luckesi, C. C. (1994). Filosofia da Educação. São Paulo: Cortez Editora.

Marins., et al. (2014). Acesso 11 de outubro de 2019, Recuperado de http://www.epsjv.fiocruz.br/sites/default/files/seguranca_alimentar_vigilancia_0.pdf

Marques, S. B., Maluf, R. S., \& Menezes, F. (1998). Caderno de Segurança Alimentar.

Moreira, M. A. (2018). Acesso 11 de outubro de 2019, Recuperado de http://www.mnpefsorocaba.ufscar.br/produtos/o-produto-educacional-por-marco-antonio moreira.

Moreira, M. C. A., Rôças, G., Pereira, M. V., \& Anjos, M. B. (2017). A interdisciplinaridade em produtos educacionais de um Mestrado Profissional em Ensino de Ciências. X Congreso Internacional sobre Investigación en Didáctica de las Ciencias. Enseñanza de las Ciencias. Sevilla, n extraordinário, 2559-2564.

Neves, D. P. (1995). Parasitologia Humana. 9a Ed., Editora Atheneu.

Nitzke., et al. (2012). Acesso 19 de outubro de 2019, Recuperado de http://www.scielo.br/pdf/bjft/v15nspe/aop_bjft_15e0102.pdf

Pereira, A. L. F. (2003). Acesso 19 de outubro de 2019, Recuperado de https://www.scielosp.org/scielo.php?pid=S0102311X2003000500031\&script=sci_arttext\&tln $\mathrm{g}=\mathrm{es}$

Pereira, A. S., et al. (2018). Metodologia da pesquisa científica. [e-book].Santa Maria. Ed. UAB/NTE/UFSM. Disponível em: https://repositorio.ufsm.br/bitstream/handle/1/15824/Lic_ Computacao_Metodologia-Pesquisa-Cientifica.pdf?sequence=1 
Porto-Gonçalvez, C. W. (2011). A globalização da natureza e a natureza da globalização. Rio de Janeiro: Civilização Brasileira.

Riquena, R. (2013). Metodologia do Trabalho Científico, Manaus, AM: Sodecam.

Santos, R. F. (2012). Recuperado de http://www.educacaopublica .rj.gov.br/biblioteca/educacao/0327.html

Saviani, D. (1985). Escola e Democracia. 6a Ed. São Paulo: Cortez Editora.

Tibola, C. S., \& Santi, A. (2017). Segurança alimentar x Segurança de Alimentos. Revista Plantio Direto.

Timm, et al. (2003). Tecnologia educacional: mídias e suas linguagens. Novas Tecnologias na Educação, 1(1).

Tonéis, C. N. (2017). Recuperado de https://www.amazon.com.br/Os-games-sala-aulagamifica\%C3\%A7\%C3\%A3o-ebook/dp/B0787MV3SL

Vital, A., \& Guerra, A. (2017). Produtos educacionais elaborados no Mestrado Profissional em Ensino: uma reflexão sobre a distância entre pesquisa e a prática docente. XI Encontro Nacional de Pesquisa em Educação em Ciências, Florianópolis: UFSC.

\section{Porcentagem de contribuição de cada autor no manuscrito}

Érica Silva Gonçalves Cabral - 80\%

Luís Gomes de Moura Neto - 20\% 